\title{
Urinary prostanoids in preschool wheeze
}

\author{
To the Editor:
}

Acute episodes of wheeze in children of preschool age are frequently triggered by viral upper respiratory tract infections and result in a significant burden to health services [1]. However, to date, the inflammatory mechanisms underlying preschool wheeze remain unclear. Mediators that have not been studied in preschool wheeze, but are implicated in the pathogenesis of wheeze in adults with asthma, include the pro-inflammatory prostanoid prostaglandin $\mathrm{D}_{2}\left(\mathrm{PGD}_{2}\right)$ [2] and the anti-inflammatory prostanoid $\mathrm{PGE}_{2}[3,4]$. In this study, we sought evidence for either increased $\mathrm{PGD}_{2}$ biosynthesis or reduced $\mathrm{PGE}_{2}$ biosynthesis, or a combination of both in children with preschool wheeze. To achieve this, we measured the major metabolites of $\mathrm{PGD}_{2}$ and $\mathrm{PGE}_{2}$ in the urine: $9 \alpha$-hydroxy-11,15-dioxo-2,3,4,5tetranor-prostan-1,20-dioic acid (tetranor-PGDM) and 9,15-dioxo-11 $\alpha$-hydroxy-13,14-dihydro-2,3,4,5tetranor-prostan-1,20-dioic acid (tetranor-PGEM), respectively $[5,6]$.

Preschool children with a history suggestive of ongoing wheeze were recruited from the Wheeze and Intermittent Treatment trial. Urine samples for prostanoid analysis were obtained from children while asymptomatic, after informed parental consent, and before the issue of trial medication (UK National Health Service Multicenter Research Ethics Committee reference 09/H1102/110). Children were aged between 10 months and 5 years, with a history of two or more episodes of wheeze, at least one of which was physician-confirmed, and at least one of which had occurred within the preceding 3 months [7]. Urine was obtained between $09: 00 \mathrm{~h}$ and 16:00 h. Healthy controls were preschool siblings of children attending the outpatient clinics of the Royal London Hospital (London, UK), and preschool children with atopic disease were recruited from a paediatric allergy clinic with a clinical diagnosis of food allergy, but with no history of wheeze (atopic disease controls).

Urine was collected and stored at $-80^{\circ} \mathrm{C}$ within $1 \mathrm{~h}$ of collection. Urinary tetranor-PGDM and tetranor-PGEM were analysed using high-performance liquid chromatography (HPLC) separation and mass spectrometry measurements. After thawing on ice, samples were centrifuged for $10 \mathrm{~min}$ at $10000 \times \mathrm{g}$ at $4^{\circ} \mathrm{C}$ and $0.5 \mathrm{~mL}$ of supernatant was used for extraction and analysis of tetranor-PGDM and tetranor-PGEM. Chemically identical internal deuterated standards were added to each sample: $10 \mathrm{ng}$ tetranor-prostaglandin $\mathrm{E}$ metabolite- $\mathrm{d}_{6}$ (tetranor-PGEM-d $\mathrm{d}_{6}$ or 11R-hydroxy-9,15-dioxo-13,14-dihydro-2,3,4,5-tetranor-prostan-17,17',18,18' 19 ,19' ${ }^{\prime} \mathrm{d}_{6^{-}}$ 1,20-dioic acid) and $10 \mathrm{ng}$ tetranor-prostaglandin D metabolite- $\mathrm{d}_{6}$ (tetranor-PGDM- $\mathrm{d}_{6}$ or 9R-hydroxy-11,15dioxo-13,14-dihydro-2,3,4,5-tetranor-prostan-17,17',18,18',19,19' $-\mathrm{d}_{6}-1,20$-dioic acid) (Cayman Chemical Co., Ann Arbor, MI, USA). Samples were then acidified ( $\mathrm{pH} 3.5$ ) using acetic acid and mixed with $0.5 \mathrm{~mL}$ tertbutyl-ether:methanol $(80: 20 \mathrm{v} / \mathrm{v})$. The organic phase of the resulting mixture was then separated by a short centrifugation step and then dried under nitrogen at $37^{\circ} \mathrm{C}$. The dried solid extract was redissolved in methanol $(60 \mu \mathrm{L}) .10 \mu \mathrm{L}$ of this mixture was used for HPLC separation and mass spectrometry measurements (Shimadzu Sil-2-AC; Shimadzu Scientific Instruments, Columbia, MD, USA) equipped with Phenomenex Synergy Fusion RP-100A $100 \times 2 \mathrm{~mm}$ column (Phenomenex International, Torance, CA, USA). The retention times for tetranor-PGEM and tetranor-PGDM were 13.2 and $13.4 \mathrm{~min}$, respectively. Analytes were measured using multiple reaction monitoring mode (MRM) tandem mass spectrometry (Qtrap 4000; AB Sciex, Concord, ON, Canada) equipped with electrospray ion source and operating in negative ionisation mode. Both tetranorPGEM and tetranor-PGDM had the same pseudomolecular ions $327 \mathrm{Mz}$ and monitored ions $309 \mathrm{Mz}$ (333 and $315 \mathrm{Mz}$ for deuterated standards). Quantification was performed using a stable isotope dilution method from the area under the peak. Urinary creatinine was assessed using a standard analytical assay and Vitros 350 (Ortho Diagnostics, Raritan, NJ, USA) and prostanoids were indexed to urinary creatinine ( $\mathrm{pg} \cdot \mathrm{mg}^{-1}$ creatinine). Urinary cotinine was determined using a commercial microplate enzyme immunoassay (Cozart Forensic Microplate; Concateno, Abingdon, UK) and exposure to environmental tobacco smoke was classified as present if the creatinine corrected cotinine concentration was $>30 \mathrm{ng} \cdot \mathrm{mg}^{-1}[8,9]$.

@ERSpublications

Tetranor PGDM, a urinary metabolite of prostaglandin $\mathrm{D}_{2}$, is increased in children with preschool wheeze http://ow.ly/Ynjy305ZY9L

Cite this article as: Grigg J, Whitehouse A, Pandya $\mathrm{H}$, et al. Urinary prostanoids in preschool wheeze. Eur Respir J 2017; 49: 1601390 [https://doi.org/10.1183/13993003.01390-2016]. 
For analysis, we divided children with preschool wheeze into two groups; those recruited at the same site as the healthy controls (group 1) and those recruited at other sites (group 2). Urinary prostanoid concentrations were $\log _{10}$ transformed before analysis. Groups were compared using either ANOVA with post hoc Tukey's multiple comparisons test or by t-test using GraphPad Prism (version 6.00; GraphPad Software, La Jolla, California, USA). Correlations (Pearson correlation coefficient (r)) and multiple regression analyses were performed using $\mathrm{R}$ (version 3.2; $\mathrm{R}$ Core Team, Vienna, Austria). Data are summarised as mean \pm SEM. A p-value of $<0.05$ was considered significant.

We recruited 24 healthy controls, five nonwheezy children with atopic disease, 149 children with preschool wheeze recruited at the same site as controls (group 1) and 810 children with preschool wheeze recruited from other sites (group 2). No child had received nonsteroidal anti-inflammatory therapy in the 2 weeks prior to urine sampling. There was no difference in age between controls and both preschool wheeze groups (healthy controls 3.0 \pm 0.27 years, atopic disease $2.9 \pm 0.81$ years, group $13.0 \pm 0.08$ years and group $22.6 \pm 0.04$ years).

There was no difference in tetranor-PGDM between healthy controls and children with atopic disease and no history of wheeze $\left(3.8 \pm 0.09 \mathrm{pg} \cdot \mathrm{mg}^{-1}\right.$ creatinine, $\mathrm{n}=24$ versus $3.8 \pm 0.06 \mathrm{pg} \cdot \mathrm{mg}^{-1}$ creatinine, $\left.\mathrm{n}=5\right)$. Tetranor-PGDM was increased in both preschool wheeze groups compared with healthy controls (group 1 $4.3 \pm 0.04 \mathrm{pg} \cdot \mathrm{mg}^{-1}$ creatinine, group $24.3 \pm 0.01 \mathrm{pg} \cdot \mathrm{mg}^{-1}$ creatinine, healthy controls $3.8 \pm 0.09 \mathrm{pg} \cdot \mathrm{mg}^{-1}$ creatinine, $\mathrm{n}=24 ; \mathrm{p}<0.0001$ ) (figure $1 \mathrm{a}$ ). In a multiple regression for PGDM including age and preschool wheeze status (group 1 and 2 combined and controls), both age and preschool wheeze status remained statistically significant $\left(R^{2} 0.13\right.$ ) (coefficient \pm SE of age $-0.096 \pm 0.01, p<0.0001$; coefficient \pm SE of no wheeze (wheeze set as reference level) $-0.37 \pm 0.75, \mathrm{p}<0.0001$ ).

In 959 children with preschool wheeze (i.e. groups 1 and 2 combined), the correlation between tetranorPGDM and age was negative $(\mathrm{r}-0.30, \mathrm{p}<0.0001)$, and PGDM was reduced in those receiving inhaled corticosteroids (ICS) $(\mathrm{p}<0.05)$. Sex, clinical pattern of wheeze (exclusive viral wheeze versus multiple trigger wheeze), exposure to environmental tobacco smoke (either parent-reported or measured by urinary cotinine) and eczema were not associated with tetranor-PGDM. In a multiple regression analysis limited to children with preschool wheeze, and including age and ICS, only age remained statistically significant $\left(\mathrm{R}^{2} 0.11\right)$ (coefficient \pm SE for age $-0.049 \pm 0.005, \mathrm{p}<0.0001$; coefficient \pm SE for ICS $0.020 \pm 0.025, \mathrm{p}=0.42$ ).

There was no difference in tetranor-PGEM between healthy controls and children with atopic disease $\left(4.4 \pm 0.07 \mathrm{pg} \cdot \mathrm{mg}^{-1}\right.$ creatinine, $\mathrm{n}=24$ versus $4.1 \pm 0.12 \mathrm{pg} \cdot \mathrm{mg}^{-1}$ creatinine, $\left.\mathrm{n}=5\right)$. There was no difference in tetranor-PGEM between controls $\left(4.4 \pm 0.07 \mathrm{pg} \cdot \mathrm{mg}^{-1}\right.$ creatinine), and preschool wheeze groups 1 $\left(4.4 \pm 0.03 \mathrm{pg} \cdot \mathrm{mg}^{-1}\right.$ creatinine) and $2\left(4 \pm 0.01 \mathrm{pg} \cdot \mathrm{mg}^{-1}\right.$ creatinine) (figure $\left.1 \mathrm{~b}\right)$. In 959 children with preschool wheeze, tetranor-PGEM was inversely associated with age $(\mathrm{r}-0.33, \mathrm{p}<0.0001)$.

These results suggest that in children with a history of severe preschool wheeze but with no active wheeze on the day of sampling, $\mathrm{PGD}_{2}$ biosynthesis, but not $\mathrm{PGE}_{2}$ biosynthesis, is increased. The mechanism whereby

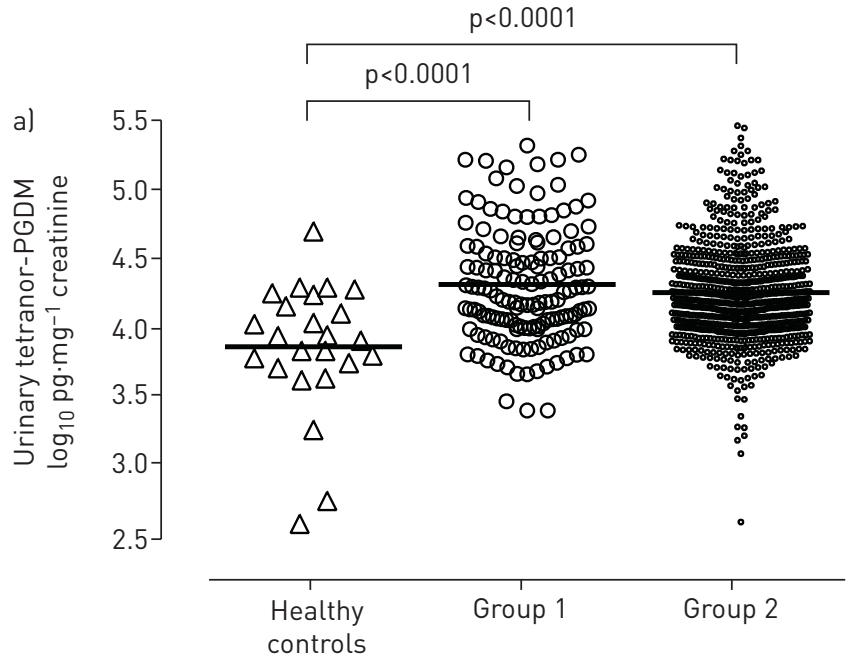

Preschool wheeze

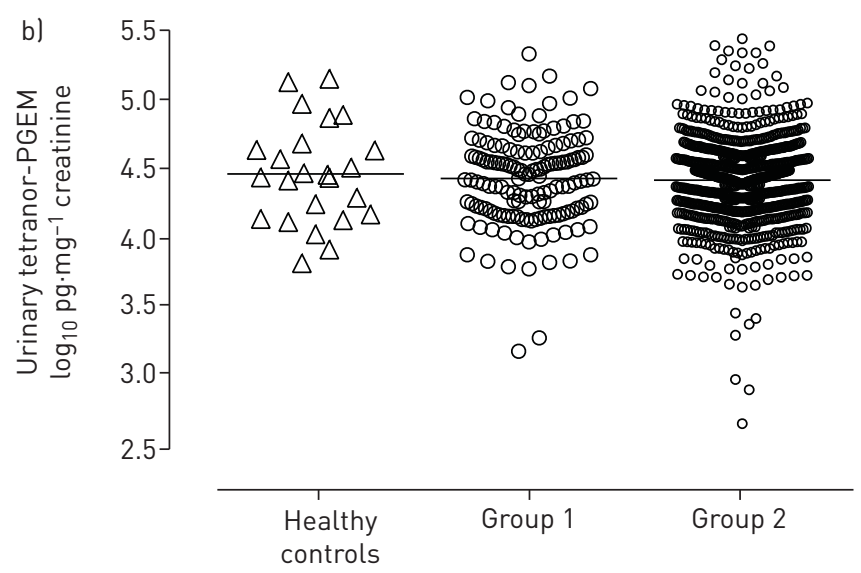

Preschool wheeze

FIGURE 1 a) Dotplot of urinary tetranor-PGDM ( $9 \alpha$-hydrox-11,15-dioxo-2,3,4,5-tetranor-prostan-1,20-dioic acid) (log 10 ) in healthy controls and children with preschool wheeze recruited at the same site as controls (group 1), and those recruited at other sites (group 2). Urinary tetranorPGDM is increased in group 1 and group 2 compared with controls (ANOVA and post hoc Tukey's multiple comparisons test). b) Dotplot of urinary tetranor PGEM (9,15-dioxo-11 $\alpha$-hydroxy-13,14-dihydro-2,3,4,5-tetranor-prostan-1,20-dioic acid) in healthy controls and group 1 and group 2 children. There is no difference between groups by ANOVA. 
airway $\mathrm{PGD}_{2}$ contributes to the pathogenesis of preschool wheeze is unclear. One potential mechanism is that increased airway $\mathrm{PGD}_{2}$, rather than directly causing bronchoconstriction, primes the airway for an exaggerated inflammatory response during viral colds, an interaction observed in an animal model [10].

There are important limitations to this study. First, although the pattern of urinary prostanoids in preschool wheeze is similar to that reported for adults with mild intermittent wheeze [11], whether increased tetranor$\mathrm{PGDM}$ in the urine reflects either increased levels of $\mathrm{PGD}_{2}$ in the airway, or increased biosynthesis in other organs is unclear. Second, we did not assess several important potential confounders of PGDM in children with preschool wheeze. For example, atopic status (measured either using skin prick testing, or specific serum IgE) was not assessed. While the nonsignificant difference in PGDM between healthy controls and controls with atopic disease is compatible with a lack of effect of atopy, the very small number of children with atopic disease means that a confounding effect of atopy remains possible. However, we are able to exclude an effect of differences in urine sampling handling since there was no difference in the urinary prostanoid profile between children with preschool wheeze recruited at the same site as controls (group 1) and those recruited at other sites (group 2).

We conclude that PGDM is a marker of potential interest in preschool wheeze, but further studies are required in better defined populations. If airway $\mathrm{PGD}_{2}$ is indeed increased in preschool wheeze, trials of new therapeutic options for this common condition would be suggested, for example of the new oral CHTR2 antagonists, which block the action of $\mathrm{PGD}_{2}$ on airway cells [12].

Jonathan Grigg $\odot^{1}$, Abigail Whitehouse ${ }^{1}$, Hitesh Pandya $^{2}$, Stephen Turner ${ }^{3}$, Christopher J. Griffiths ${ }^{1}$, Tom Vulliamy ${ }^{1}$, Robert T. Walton ${ }^{1}$, David B. Price $\odot^{3}$, Marek Sanak ${ }^{4}$, John W. Holloway ${ }^{5}$, Lee Noimark ${ }^{1}$, Maia Lesosky ${ }^{6}$, Rossa Brugha ${ }^{1}$, Lee Koh $^{1}$ and Chinedu Nwokoro ${ }^{1}$

${ }^{1}$ MRC and Asthma UK Centre in Allergic Mechanisms of Asthma, Blizard Institute, Queen Mary University of London, London, UK. ${ }^{2}$ Dept of Infection, Immunity and Inflammation, University of Leicester, Leicester, UK. ${ }^{3}$ Division of Applied Health Sciences, University of Aberdeen, Aberdeen, UK. ${ }^{4}$ Dept of Medicine, Jagiellonian University Medical School, Krakow, Poland. ${ }^{5}$ Human Development and Health, University of Southampton, Southampton General Hospital, Southampton, UK. ${ }^{6}$ Division of Epidemiology and Biostatistics, School of Public Health and Family Medicine, University of Cape Town, Cape Town, South Africa.

Correspondence: Jonathan Grigg, Blizard Institute, Queen Mary University of London, 4 Newark Street, London, E1 2AT, UK. E-mail: j.grigg@qmul.ac.uk

Received: July 122016 | Accepted after revision: Oct 312016

Clinical trial: This study is registered at ClinicalTrials.gov with identifier number NCT01142505.

Support statement: Funding was received from the UK National Institute for Healthcare Research, Efficacy and Mechanisms Evaluation (NIHR-EME) Programme (reference 08/43/03). J. Grigg is part-funded by the NIHR North Thames Collaboration for Leadership in Applied Health Research and Care. Funding information for this article has been deposited with the Open Funder Registry.

Conflict of interest: Disclosures can be found alongside this article at erj.ersjournals.com

Acknowledgements: The author contributions are as follows. J. Grigg was the chief investigator, planned and provided overall supervision of the study, wrote the manuscript and vouches for these data. A. Whitehouse performed data analysis and contributed to the final manuscript. M. Lesosky undertook the multiple regression analysis and contributed to the final manuscript. C. Nwokoro and R. Brugha supervised the study and contributed to the final manuscript. H. Pandya, S. Turner, R. Walton and D. Price contributed to study planning and to drafting the final manuscript. T. Vulliamy, J.W. Holloway and C.J. Griffiths contributed to study planning and contributed to the final manuscript. M. Sanak performed urinary prostanoid analysis and contributed to the final manuscript. L. Noimark obtained data from controls and contributed to the final manuscript. L. Koh supervised data from controls, collated data and contributed to the final manuscript.

\section{References}

1 Davies G, Paton JY, Beaton SJ, et al. Children admitted with acute wheeze/asthma during November 1998-2005: a national UK audit. Arch Dis Child 2008; 93: 952-958.

2 Hirai H, Tanaka K, Yoshie O, et al. Prostaglandin D2 selectively induces chemotaxis in T helper type 2 cells, eosinophils, and basophils via seven-transmembrane receptor CRTH2. J Exp Med 2001; 193: 255-261.

3 Profita M, Sala A, Bonanno A, et al. Increased prostaglandin E2 concentrations and cyclooxygenase-2 expression in asthmatic subjects with sputum eosinophilia. J Allergy Clin Immunol 2003; 112: 709-716.

4 Aggarwal S, Moodley YP, Thompson PJ, et al. Prostaglandin E2 and cysteinyl leukotriene concentrations in sputum: association with asthma severity and eosinophilic inflammation. Clin Exp Allergy 2010; 40: 85-93.

5 Zhang Y, Zhang G, Clarke PA, et al. Simultaneous and high-throughput quantitation of urinary tetranor PGDM and tetranor PGEM by online SPE-LC-MS/MS as inflammatory biomarkers. J Mass Spectrom 2011; 46: 705-711.

6 Song WL, Wang M, Ricciotti E, et al. Tetranor PGDM, an abundant urinary metabolite reflects biosynthesis of prostaglandin D2 in mice and humans. J Biol Chem 2008; 283: 1179-1188.

7 Nwokoro C, Pandya H, Turner S, et al. Intermittent montelukast in children aged 10 months to 5 years with wheeze (WAIT trial): a multicentre, randomised, placebo-controlled trial. Lancet Respir Med 2014; 2: 796-803.

8 Henderson FW, Reid HF, Morris R, et al. Home air nicotine levels and urinary cotinine excretion in preschool children. Am Rev Respir Dis 1989; 140: 197-201. 
9 Ehrlich R, Kattan M, Godbold J, et al. Childhood asthma and passive smoking. Urinary cotinine as a biomarker of exposure. Am Rev Respir Dis 1992; 145: 594-599.

10 Shiraishi Y, Asano K, Niimi K, et al. Cyclooxygenase-2/prostaglandin D2/CRTH2 pathway mediates doublestranded RNA-induced enhancement of allergic airway inflammation. J Immunol 2008; 180: 541-549.

11 Daham K, Song WL, Lawson JA, et al. Effects of celecoxib on major prostaglandins in asthma. Clin Exp Allergy 2011; 41: 36-45.

12 Hall IP, Fowler AV, Gupta A, et al. Efficacy of BI 671800, an oral CRTH2 antagonist, in poorly controlled asthma as sole controller and in the presence of inhaled corticosteroid treatment. Pulm Pharmacol Ther 2015; 32: 37-44.

Copyright (CERS 2017 